\title{
Some further considerations on the Supports of the Female Pelvic Viscera, in which the Intra- Abdominal Pressure is still further defined.*
}

\author{
lyy R. II. Paramore, M.D. (Lond-), F.R.C.S. (Eng.).
}

THE position of the female pelvic viscera and the maintenance of this position in the pelvis is the result of the equilibrium of two sets of forces, which simultaneously appear and oppose and paralyse each other: the one acts from above and tends to thrust the viscera downwards towards the pelvic outlet; the other acts from below, opposes the first and tends to prevent a descent of the viscera. The first force is known as the intra-abdominal pressure; the second force is the resistance resulting from the tonic contraction of the levator ani muscle.

These opposing forces, like all other vital forces, are during health continually undergoing variations in magnitude, but these variations are uniform, symmetrical, counterbalancing; one does not increase without similar increase of the other, but both increase together, reach the climax together, remain sustained together, together diminish or perhaps, as in sleep, disappear together. That is to say, they are co-ordinated. This uniformity of action is but the result of the synchronous and synergetic contractions of two sets of muscles, which play the parts of antagonists to each other; for the intraabdominal pressure is nothing other than the result of the simultaneous contraction of all the muscles of the abdomino-pelvic walls. This uniformity, which is determined by nervous control, even persists when the pelvic floor musculature has to permit the functioning of the struetures which pass through it. On bearing down and during defacation the pelvic floor musculature descends and occupies a lower position than usual. It still, however, remains contracted and still supports the viscera. In this lowered position of the floor it is plain the contained viscera will come to occupy a lower place. During the excursion of this visceral descent the visceral pelvic connective-tissue comes into action and plays a part in preventing a slipping downward of the cervix. It has been supposed that this visceral connective-tissue plays a greater part than this, but reasons have already been advanced to show that this conception cannot be accepted..$^{1}$

* Amplified from a paper read before the Anatomical Section of the British Medical Association at Sheffield, July, 1908.

1. See recent paper by the author, "The Supports-in-Chief of the Female Pelvic Viscera." Proc. Royal Soc. Med., June, 1908, and Journ. Obstet. and Gynacol. Brit. Emp., June, 1908. 
All the viscera in the female pelvis, the bladder, the uterus, the upper (i.e., intra-pelvic) part of the vagina, the ovaries and Fallopian tubes, as well as the rectum, are supported and maintained in their normal position by the same means, for they are all subject to the same physical laws; but it is simpler to concentrate our attention upon the position of the uterus and to consider how it is maintained in its position, always remembering that this is but an index of how the other viscera are supported.

The forces, which either pull or thrust the uterus downwards, are (1) the action of gravity upon the uterus; $(2)$ the weight of the superimposed viscera; and (3) the intra-abdominal pressure. The weight of the normal uterus is about $1 \frac{1}{2}$ ounces. What the pressure upon the superior surface of the anteverted uterus, as a result of the mere weight of the superimposed abdominal viscera, is, I do not know; but Prof. Keith states that in the erect posture there is a positive pressure of 20 to $28 \mathrm{~mm}$. of $\mathrm{Hg}$. in the rectum, whilst the measurement in the stomach shows a pressure of 6 to $12 \mathrm{~mm}$. Hg., and, further, that this increase of pressure in the rectum over and above that in the stomach is due to the weight of the superimposed viscera. ${ }^{1}$ Therefore the effect of gravity upon the uterus, i.e., the weight of the uterus plus the weight of the superimposed viscera acting upon it, amounts on on the average to a pressure of about $13 \mathrm{~mm}$. Hg. This is such a small force that it may, for the present purpose, be neglected.

The intra-abdominal pressure, on the other hand, may easily reach as much as 90 to $120 \mathrm{~mm}$. Hg.; and this is not a rare but a very usual and frequently recurring increase. Moreover, these rises are often produced with extreme suddenness, as on coughing and sneezing, which, of course, necessitates an equal rapidity in the production of an increased resistance below if the pelvic viscera are to be preserved in their positions and prevented from extrusion. It is evident that the alterations in the magnitude of this force, acting upwards from below, which, it must be allowed, are necessary to maintain equilibrium in all the multitudinous pressure relations of the abdomino-pelvic cavity, besides being considerable, must be capable of frequent recurrence and rapid induction. Are conditions such as these to be confidently attributed to connective-tissue? Is it possible to conceive that connective-tissue, such as the perivascular tissue, can fulfil such requirements, a tissue which is anything but elastic except to a vory trifling and negligible extent, and which in all the movements of the body plays but a passive part? Is it supposed that when a woman bears down and the pelvic floor is seen to descend that a return to the normal level is the result of some strange and unknown activity in this connective-tissue? or how do

1: Article on Enteroptosis, System of Medicine, by Allbutt and Rolleston, 1907, iii, p. 860 . 
the supporters of the hypothesis in its farour suggest the pelvic Hoor returns? And when the floor does not descend what is the mechanism which prevents this descent? Can the connective-tissue at one time allow descent and at another time prevent descent? Has if indeed the function, although it has not the structure, of contractile tissue? Does it react to the will? and is its activity dependent upon nervous supply?-in connection with which note well the significance of spina bifida in association with congenital prolapse. But these criticisms remain unanswered, nor is this mighty stumbling block of 'congenital prolapse' explained by the adherents of the connectivetissue hypothesis. There is no doubt that the exceedingly variable and often excessively raised pressures within the pelvis can only be met by means similar to those which produce these changes, and can only be obtained by the use of a highly elastic and contractile material; and consequently we find the same means (muscle) employed to close in the pelyis as exists above in the abulominal walls, which play the chief part in the production of these changes.

But because these variations of pressure within the abdominal cavity have been overlooked, and because the levator ani muscle has been considered a degenerated muscle and the parametric tissue is known to be fairly strong and tough, it has been supposed that this visceral connective-tissue of the pelvis is the chief and only support of the uterus. Whilst it is true that the levator ani muscle has degenerated phylogenetically, in that man has lost the tail, it is no less true that evolutionary changes, just the reverse of degenerative ones, have also beex steadily in progress and that ontogenetically the levator ani, in the healthy and sound condition, cannot be eonsidered a degenerated muscle. ${ }^{1}$ As regards the visceral connective-tissue of the pelvis, it were indeed possible for this tissue to maintain the uterus in its position if it had merely to bear the weight of the uterus and if the pressure from above were always a negligible one; but how ean this connective-tissue meet variations of such magnitude as occur in the normal pressure relations within the pelvis? Although the visceral connective-tissue is strong and thick in places it is not sufficiently strong for this purpose.

What, however, is the cause of these pressure variations within the abdomino-pelvie cavity? There is no actual cavity within the peritoneal sac, for the peritoneal coverings are everywhere in contact with each other. This contiguity of surfaces is the result, not of a negative pressure as obtains within the pleural sacs, but of a positive pressure, the consequence of the pressure exerted upon the contained viscera by the tonic contraction of the muscular walls. That is to say, the abdomen, of which the pelvis may be considered a recess, is packer full of viscera. If we pack a box full with articles, we know

1. See Author's paper, already referred to. 
that the closer these are placed to each other the less likelihood is there of their breaking loose within the box during a supposed transit, and the less likelihood of their being damaged. If all the articles are made of elastic tissue so that they can be squeezed more easily closely together, the certainty of their remaining well packed is assured-for if the lid yields the contents, in virtue of thcir elasticity, expand and still press against each other. If a small india-rubber bag, containing a small quantity of air, is placed among the other elastic articles and is connected with a manometer, then, when the lid of the box is thrust down upon its contents and thus is made to exert pressure upon them, the pressure within the box will rise as will be shown by the manometer. This pressure measurement is taken as the pressure within that part of the box where the bag has been placed. It is tho result not only of the articles pressing against each other but also against the sides, the bottom and the lid of the box. Is it not correct to say that this pressure within the box maintains the several articles in their positions? not by itself, of course, for if we take the sides of the box away the pressure within disappears and the position of the previously contained articles becomes disturbed and remains unassured. It is the combination of the pressure within and the resistance of the enclosing walls, which resistance itself is the cause of the internal pressure, both acting concomitantly and in opposition to each other with a resulting equilibrium, which determines the security of the several positions of the contained articles. If the internal pressure disappears, e.g., by the removal of one side of the box, which will, of course, entail an alteration in the positions of the articles, equilibrium will again subsequently be established; but with this difference that the maintenance of the altered position of any one article will not be secured, but its position will be subject to any extrinsic force which, by accident, may chance to fall upon it.

If now in this box, one side instead of wood is made of rubber, it is clear the conditions within the box will be better adapted to allow of any increase or any diminution in volume of the compressed articles within the box occurring, and more suitable for pressure variations being exerted upon the contents from without.

If, instead of a box, we have a cavity formed by a framework of bones, united to each other by joints, ligaments and muscles, and which itself is therefore capable of a considerable amount of variation in capacity, from changes in the relative positions of its several parts; and which is completed by a system of highly elastic sheets of muscle on all sides, it is clear that the cavity is highly adapted, in virtue of variations in the tension of the enclosing muscles, for changes in volume and therefore of pressure within.

These variations of pressure, which have been actually shown to occur experimentally and have been measurcd by various observers, 
are produced by variations in the position of the skeleton and in the tension or contraction of the muscular enclosing walls. If the capacity of the cavity is small, as for example, when the spine is flexed and the sternum has approached to the pubes, as occurs usually at stool, or as obtains in kyphosis, scoliosis, and in the lowered position of the diaphragm in chronic bronchitis and emphysema of the lungs; or if the contents of the cavity, that is the volume of the contained viscera with their contents and their mesenteries, is relatively large (distended abdomen); then since the musculature of the body is always in a state of tonus, it is clear the pressure within the abdomino-pelvic cavity will always be positive, that is greater than the atmospheric pressure. That this is so in ordinary health is shown by the figures quoted-a positive pressure of 20 to $28 \mathrm{~mm}$. Hg. in the rectum, and 6 to $12 \mathrm{~mm}$. Hg. in the stomach in the erect posture at rest; when movements occur, especially violent ones, the pressure immediately rises and may easily reach 90 to $120 \mathrm{~mm}$. $\mathrm{Hg}$. With the individual lying down on the back at rest, the pressure immediately beneath the anterior abdominal wall will approach or even reach zero-depending on the tone of the musculature; if this tonus disappears (e.g., anæsthesia), zero will in this place be reached. Even in this position it is impossible for a negative pressure to arise, for if this were to occur the lax anterior abdominal wall would sink in until the pressure within and without would equal the atmospheric pressure and a measurement would show a reading of zero.

If the abdomen is carinated, i.e., the abdominal contents are relatively small, if the spine is extended, the base of the thorax expanded and projected anteriorly so as to cause an increase in the capacity of the abdomino-pelvic cavity, and if at this time the recti abdominis muscles, which in a carinated abdomen pass in a curred course first downwards and backwards to the lumbar spine and then downwards and forwards to the pubes, are strongly contracted, they will tend to occupy a more anterior position, in which they will pass from the thorax to the pubes in a straight, i.e., shorter line, rather than in a curved and longer one. This will cause a forward movement of the linea alba and tend to reduce the pressure within this kind of abdomen; and Prof. Keith was actually able in this way to obtain a small negative pressure within the stomach cavity, i.e., in the upper part of the abdomen. ${ }^{1}$ But I do not know that a negative

1. Prof. Keith writes $(i b i d)$ : "There is always a positive pressure in the uncontracted stomach, which occupies the left dome of the diaphragm. Moritz and Schwerdt, Kelling, and the writer, independently of these observers, estimate that the intra-gastric pressure when the stomach is passive is 4 to $8 \mathrm{~mm}$. Hg. when standing upright during expiration, and rises to $12 \mathrm{~mm}$. $\mathrm{Hg}$. during inspirationthat is to say, the diaphragm is always in a state of tone or contraction, exerting pressure on the liver, stomach and abdominal contents. Schreiber, on the other hand, found a negative intra-gastric pressure of 5 to $1.5 \mathrm{~mm}$. Hg. By taking a deep 
pressure bas ever been found, in the erect posture, to exist in the rectum, nor can we cxpect this to occur whilst the pressure in the uncontracted stomach is positive. And we must also remember that whilst the forward movement of the recti abdominis muscles is tending to oceur, the other muscles of the abdomino-pelvic cavity, the transversalis abdominis and the oblique muscles, are also contracting. These, encircling the abdomen, the former horizontally, the latter obliquely, exert pressure inwards upon the flanks and thus tend to maintain a positive pressure within and in this way support the kidney. From these considerations we must assume, until the contrary is proved, that in the erect posture the pressure within the polvis is always positive; and we know that on active and much more on riolent movement it is considerably so. In view of these theoretical deductions and the findings of Prof. Keith and other observers, it is impossible to understand how the atmospheric pressure, acting from below, can play a part in the maintenance of the polvic visceral position, as has recently been again brought forward. Further, wo must also remember that this same atmosphoric pressure exerts tho same force upon that part of the lower abdominal wall which is in relation to the inlet of the pelvis; and that therefore these two, the one acting upwards from below upon the pelvic foor, and the other acting from in front upon the lower abdominal wall, would balance one another; so that if the viscera were pressed downwards by the powerful abdominal muscles plus the atmospheric pressure upon the belly wall and were only supported from below by the atmospheric pressure, it is certain a prolapse of the viscera would occur. Besides, the coils of small intestine in the pelvis and often the pelvic colon and the rectum contain gas, certainly usually not lower, and often much higher than the external pressure. If in these cases the visceral support were dependent upon atmospheric prossure, a prolapse shonld occur each time an excessive pressure from any cause whatsoever appeared within. But we know that such a prolapse docs not occur; nor can we suppose that the level of the uterus is to he estimated by barometric observations, nor

thoracic breath, and holding the abdominal walls as rigid as possible, $\mathrm{I}$ found it possible to produce a negative intra-gastric pressure of $4 \mathrm{~mm}$. $\mathrm{Hg}$. This is indicated also by the retraction of the epigastrium. The intra-gastric pressure in enteroptosis has not, as far as I know, been investigated, but there can be littlo doubt that in the standing posture a negative pressure will be found, at least in the early stages; for the epigastrium becomes drawn inwards in such cases to fill up the vacuum. It is just possible that Schreiber's observation was made on an individual with a tendency to ptosis. In cases of enteroptosis Schwerdt found that the intra-abdominal pressure,

1. That "whilst the vagina was a clased passage the pressure of the atmosphere on the pelvic foor was an important factor in maintaining proper support of the superimposed viscera. ..." Prof. Thompsom. Proc. Royal Soc. Med., Obstet. and Gyn. Sec., June, 1908 , p. 220. 
think that prolapse is the result of altered climatic conditions (e.g., balloon or mountain ascent), as must indeed be the case, if this factor is of the importance it has been stated to be.

During active life, therefore, and certainly in the erect posture of the trunk, the intra-abdominal pressure is always thrusting the viscera downwards; and it must therefore be considered as playing a part, indeod a principal part, in the maintenance of the visceral position. If, as a result of the recumbent position, the prossure in somo parts of the abdomino-polvic cavity falls to zero, the visceral position can no longer be dependent upon tho intra-abdominal pressure, for this has disappeared; but simultaneously with this disappearance the contractions of the enclosing muscles have also disappeared, and the viscera then lie passively resting upon each other and upon the posterior abdominal wall.

Hill and Barnard have shown the importance of a positive pressure within the abdomen for the functioning of the vascular system, ${ }^{\text {I }}$ and it may play a not unimportant rôle in connexion with the absorbent processes of the stomach and intestine. The importance, and indeed the necessity, of a raised intra-abdominal pressure in coughing and in defreation, are at once obvious; and certainly by maintaining the viscera against each other with a certain pressure, which varics with the posture of the body and the part of the abdominal cavity examined, it will keep the organs in their respective places and prevent them from sagging downwards by reason of periodic increase and weight of their contents, e.g., the stomach after a large meal.

Moreover, the capacity for undergoing changes in volume of the abdomino-pelvic cavity is of importance. The necessity for this is shown by the mere fact of variation in the volume of the abdominal contents resulting from ingestion of food, secretion of urinc and its

1. Thus these authors say (Hill and Barnard, on "The Influence of the Force of Gravity on the Circulation of the Blood," Journ. Physio., Ton., 1897, xxi, p. 323) : "The capacious abdominal veins are supported by the abdominal wall, which forms a muscular sphere. The tone of the muscolar wall depends on the respiratory centre. The parietal layer of the peritoneum is an extremely extensile and clastic membrane.

The purpose of our experiments is to prove that:

(1) By the support of the abdominal wall the distension of the veins under the hydrostatic stress of gravity is largely prevented.

(2) $\mathrm{B}_{y}$ expiratory contractions of the abdominal muscles the hepatic and other veins within the abdomen can be compressed and the right heart filled with blood." Gravity on the Circulation of the Blood," Journ. Plyysiol., Lon, 1897, xxi, p. 323) : "The common practice of wearing abdominal belts for weakness, and the application of binders after parturition, find a physiological explanation in the results of this research. Roy and Adami have suggested that the almost universal practice of wearing waist-belts and stays is due to the fact that compression of the abdomen increases the output of the heart and the blood supply to the brain and muscles." 
retention in the urinary bladder; as well as the more slowly produced volume changes caused by variations in the deposit of fat, and in the growth and expansion of the pregnant uterus, etc. In connexion with this temporary or permanent increase of volume of contents, the pressure as a rule is not continuously increased, because the abdominal walls accommodate themselves to the increased contents and their tension or tonus is reduced. ${ }^{2}$ Thus the pressure within is maintained on the average at a level, which is constant for the same posture and the same locality within the abdomen, and only varies within wide limits when some active or more or less violent movement has to take place. The necessity, then, for the maintenance of a more or less uniform positive intra-abdominal pressure and for an ever varying capacity, is ensured by the existence of highly elastic and rapidly reacting, striated, muscular, abdominal walls, which indeed play the largest share in the production of these pressure and volume variations. Nor, perchance, should we have to reckon with the pelvic floor musculature, which is only capable of small, though distinct, excursions in the cranio-caudal direction, and which movement, even when maximal, can have little effect by jtself in altering either the pressure or the volume of the abdomino-pelvic cavity, but for the fact that it has to undergo frequent changes to permit of defæcation and parturition. Had the evolutionary changes which resulted in the appearance of the hind fins and pelvic plates, ${ }^{2}$ and which subsequently determined their union to the vertebral column by dorsal upgrowths, had this mechanism developed behind the cloaca instead of in front of it, then it is possible that in man the pelvic floor as a movable membrane would have ceased to exist, and would have played a part in the pressure and volume changes within the abdomino-pelvic cavity in the same way as other non-yielding, osseous structures do. But the pelvic girdle has evolved in front of the cloaca, and the region of the cloaca has thus been eut off from the abdominal wall, and has developed into a highly complex part; for it has not only to participate in the functions of the abdominal wall, but also has to permit the transit of the canals of the genito-urinary and alimentary apparatus, and to play an active and especial part in connexion with their functioning. Of these especial functions, defæcation is perhaps the chief, certainly the most frequently recurring in both sexes; and it would be interesting to learn the fate of the pelvic floor musculature when it had been robbed of this function for many years in cases of occluded rectum in the male which had survived colotomy. The pelvic floor musculature also functions during the reproductive processes in both sexes; but in the female these are especially important, for it has to be capable of a twofold

1. Halban and Tandler, Anatomie und Aetiologie der Genitalprolapse beim Weibn, 1907, p. 208.

2. Wiedersheim and Parker, Comparative Anatomy of Vertebrates, 1907. 
action, first the transit of the child, and second the preservation of the pressure and volume variations of the abdomino-pelvic cavity.

'The necessity for these local functions has resulted in specialisation of structure, nor would it be possible for them to occur were the pelvic floor a rigid and unyielding diaphragm. The necessity for its elasticity for local functions being thus determined, the necessity also arose for it to participate in the pressure relations within the abdomino-pelvic cavity. This evolutionary change has been associated with the gradual assumption of the erect posture and the loss of the tail. Whilst, then, the elastic pelvic floor, because of the relative smallness of the pelvic cavity, and its limited excursion possibilities, is unable by itself to alter appreciably the pressure and volume conditions within the abdomino-pelvie eavity, on the other hand, acting with the abdominal wall muscles, it can and does, by offering a tirm resistance to pressure from above, as a result of contraction of its fibres, allow the large abdominal walls to function, at the same time as, by its own resistance, it prevents the descent of the viscera; and the simultaneous contraction of these two sets of muscles, acting synchronously and synorgetically, creates the increase of pressure within the abdomen which, known as the intra-abdominal pressure, must be recognized as playing an important part in the maintenance of the visceral position.

Even in the absence of unanswerable criticisms, considerable difficulty has been experienced in accepting this conception as faithfully portraying the state of things actually occurring in the pelvis; for it has been thought that the muscle could not for long contract and support the viscera, but that muscle fatigue would soon arise and result in a temporary paralysis, and that then the fascia of the pelvis would naturally come into play and support the viscera. Thus it has been considered that the fascia of the pelvis, which is thus conceived as sparing the muscle and preventing its fatigue, must usually support the viscera, and that it therefore is the essential element in the maintenance of the visceral position; and that the levator ani muscle is subsidiary in action and is only called upon to support and assist the fascia in extraordinary conditions, during which the strain is greater than normal.

Strange as it may seem, however, the reverse of this is actually the case, and muscle fatigue, though contraction is continuons, does not, in health, occur. It is a remarkable and wonderful fact that all the muscles of the body are always contracted, and are never in a state of complete relaxation (paralysis). Examination of the tendons in the popliteal space, or at the wrist, when the ler or the arm respectively is laid passively at rest, will convince any one that this is so. This muscle tonus is a vital phenomenon, indeed something to wonder at. It is not perhaps more wonderful that during life we should have a continuously raised muscle tension, than we should 
possess a continuously raised blood pressure; but whilst the existence of the latter is universally known, the former is, in fact, scarcely recognized. The tendons in the popliteal space and about the wrist joint, as all other tendons, including the parietal connective tissue attachments of the levator ani muscle, are actually dependent upon the continuous functioning of their respective muscles for their anatomical strength; and when fatigue occurs, if this is often repeated, not only the muscle but the whole fibro-muscular mechanism suffers and an atrophy of the whole ensues. Indeed, the connective tissue attachments of muscle are only capable of bearing a tension that the muscle is capable of producing; and when a greater tension than this arises stretching occurs, and this yielding continues until either the tension is sufficiently reduced or some other structure is come upon which shares the burden. As regards the visceral connective tissue of the pelvis, however, different conditions exist; for it does not form a means of attachment of the muscle to the bone. But virtually this connective tissue is as dependent upon the continuous functioning of the levator ani muscle as are the connective tissue attachments of the muscle itself. The truth of this is shown by the morbid anatomy of prolapse, for when the muscle gives way definite changes occur in this connective tissue in consequence of its having lost this muscular support.

In old standing cases of prolapse, we find this connective tissue has become thickened and hypertrophied, and forms, with the inverted vagina, a tough fibrous sac for the contained prolapsed uterus, bladder, rectum and intestines; and that its localized thickenings known as the parametrium, the cardinal ligaments, the utero-sacral ligaments, and the pubo-vesico-uterine ligaments, which all pass radially inwards towards the uterus from the pelvic walls, have undergone, not only a lengthening but also an hypertrophy, so that they are, as a matter of fact, much thicker and stronger than normal. This thickening of this subperitoneal tissue-for it is nothing lessis the result of the frequent impactions downwards into the pelvis of the impulses from above, the result of the variations of the intraabdominal pressure, which, when the muscle has become deficient, the connective tissue has had to meet. The intermittency of the thrusting still further downwards of the already prolapsed uterus and other viscera has resulted in an activity occurring within the subperitoneal tissue and the formation of definite fibrous tissue, which, at length, with the re-establishment of equilibrium, ${ }^{1}$ prevents the further descent of the prolapse and acts as a barrier, indeed an inelastic one, to the impulses of the lessening intra-abdominal pressure.

1. For a limit is eventually reached in the descent of the prolapse. This is due to the diminution of the down-thrust, the result of weakened abdominal muscles, and the increase of resistance from below, the result of the progressive thickening of the sac. 
Anyone who has removed a uterus which has been prolapsed for a considerable time, will, I believe, testify to this increased thickening of the sub-peritoneal tissue; and this thickening is exactly comparable to the similar thickening which occurs in the sac of any hernia of long standing, e.g., in serotal hernia. But it has been stated that " the one constant essential cause of prolapse is relaxation of the perivascular sheaths," 1 and that these are due to congenital defects, parturition and senile atrophy. ${ }^{2}$ If this is so, is it not remarkable that this connective tissue, which binds the uterus and other viscera to the pelvic walls, should, when prolapso occurs, commerice to take on actual supporting functions as is shown by a hypertrophy? If prolapse is due to a lesion of this connective tissue, should we not expect, in well-marked cases of prolapse, to discover the lesion, which is presumably a degenerative one (but of its nature this authority has not, to my knowledge, yet spoken), instead of which we find remarkably well developed, very thick and very tough fibrous tissue. I have already shown reason why the natural normal thickenings found within the sub-peritoneal tissue covering the pelvic floor exist. I believe and have said they are supporting in nature, and that they come into play whon the pelvic floor occupies a lowered position as it does during the bearing-down efforts at stool." They may also play a part in the anchoring of the cervix when the uterus is displaced by reason of local changes in the surrounding organs, as occurs on distension of the bladder and of the rectum. ${ }^{4}$ During defreation the nterus with the other viscera is thrust downwards upon the lowered pelvic floor, and this downward thrust causes lines of tension to arise within the sub-peritoneal tissue; and thus these thickenings have become developed as a result of the alternating conditions of relaxation and moderate tension within this tissue. When the muscle habitually occupies a lowered position, this subperitoneal tissue is called upon to perform yet increased work, and in consequence a further development of its tissues ensues.

These theoretical considerations then, which show that the pelvic floor must be capable (1) of accommodating itself to the pressure relations within the abdomino-polvic cavity, and (2) of meeting local requirements, are substantiated by anatomical, clinical, and pathological facts: in that (1) the pelvic outlet is shut off from the outside by a muscular sheet, (2) this muscular sheet is easily palpable in the living, and (3) that when this sheet has been sufficiently injured, and

1. Compare the action of the so-called "Suspensory Muscle of the Duodenum," Gyn. Sec., January, p. 58.

2. See paper by Author, already referred to, Proc. Royal Soc. Med., June 1908 . 1908.

3. Ibid, June, 1908, p. 218.

4. Fothergill, "Supports of Pelvic Viscera," Proc. Royal Sor. Med, Obst. and q.v. Lancet, June 20, 1908, p. 1760. 
the intra-abdominal pressure periodically becomes sufficiently high, prolapse occurs.

In spite, however, of these discoveries, and simple, straightforward and obvious as this conception is, the adherents of the visceral connective tissue hypothesis still call it in question, and criticisms have been made in respect of both the factors concerned.

\section{As regards the Pelvic Floor Musculature.}

Thus, Dr. Fothergill, in the endeavour to show the valuelessness of the levator ani muscle, asks whether anyone could suppose that the pelvic viscera, severed from all their connexions with the pelvic walls, would remain for any length of time in their usual positions within the pelvis. "A few movements of the bowels," he continues, "and they would certainly be expelled." (Discussion, Proc. R.S.M. loc. cit., p. 219.)

We have, however, only to reflect that pessaries, which have no connexion with the pelvic walls, and which lie free and even loose within the vagina, may be worn for years without involuntary removal, for the fallacy of this suggestion to become obvious. The pessary does not depend upon any ligamentous means of suspension, but it plays the part of a scaffolding, which, in the erect posture of the body, bridges over the gap intervening between the two median borders of the levator ani musele (the pubo-rectalis). When the genital fissure has become so permanently enlarged that it is not obliterated or sufficiently diminished on contraction of the muscle which forms its boundary, then a descent of whatever lies above the deficiency in the pelvic floor will occur. This consists of the anterior vaginal wall with the superimposed bladder. The increased pressure within the abdomen, consequent upon strain or bearing down (increased intra-abdominal pressure) forces the abdominal viscera downwards; these press upon the bladder, and the part least supported yields. Thus a bulging of the base of the bladder occurs, and this pushes the anterior vaginal wall before it. This is the rtiology of cystocele. If a ring pessary is inserted into the vagina and it is sufficiently large to overlap the median borders of the muscle bounding the deficiency in the pelvic floor, when the intra-abdominal pressure is increased, e.g., by coughing, the levator ani muscle contracts, supports the pessary laterally on each side, whilst upon the superior surface of this, especially upon the anterior bar of the ring, the cystocele is projected but is prevented from descent by its contact and resistance. This is how a pessary acts.

Moreover, examinations often disclose the presence of a very freely movable uterus, a uterus which, on bimanual examination, can bo moved to a considerable extent in all directions, and this in the absence of prolapse or even of symptoms of this complaint (backache, dragging pains, etc.) In such cases, the mobility of the uterus 
can only co-exist with relaxed or elongated ligaments, so that it is certain that fixation to the pelvic wall is not essential for the retention of bodies, natural or artificial, within the pelvis.

In further support of his contention, this author has even stated that "the levator ani and the external layer did not prevent the expulsion of the products of conception, nor of freal masses" (ibid).

As regards this statement, I would like to ask obstetricians, whether they agree to the first statement, and gynæcologists, whether they accept the second. Is it not a fact that the levator ani muscle frequently causes delay and sometimes prolonged delay in the second stage of labour? that indeed the obstruction it causes to the passage of the child's head gives perhaps the most frequent indication for the use of forceps? When the child's head is low in the pelvis, and does not advance even in the presence of good pains and the absence of bony obstruction, as is shown by the recession between the pains, what causes the delay? The delay is caused by the obstruction that is presented by the contracting levator ani musele (pubo-rectalis portion).

Thus Piquand and Hue write: (Revue de Gynécologie et de Chirurgie 1 bdominale, January-Fobruary, 1908, p. 10) "The manner in which the levator dilates depends principally on the development of the muscle and on the time when flexion of the head occurs. When the levator is poorly developed it easily dilates. On the other hand, when it is very powerful, it opposes an important obstacle to the passage of the head and may be a cause of dystocia."

They state that Smellie, in 1771, noted a number of such cases, which lingered from rigidity of the vagina and maternal soft parts, and that these cases occurred in women of a robust and substantial temperament, and that Smollie treated them by bleeding.

These authors continue: "In 1807 Gardien remarks that vigorous women are generally delivered more slowly and with more difficulty than others, because the parts offer more resistance and are less easily dilated. After him, Cazeaux insists on this fact and states that in strong and well-developed primiparæ it is frequent to see labour, up till now quite regular, abruptly arrested when the head meets the pelvic floor, and that it may remain thore several hours without advancing a millimetre, in spite of the violence of the uterine contractions. He thinks that the obstacle is obviously due to the contraction of the muscles of the perineum, and admits that this muscular resistance is the cause which necessitates most often the employment of forceps. This resistance of the perineum in certain women is due almost exclusively to the contraction of the levator muscle and only occurs in women who possess it well developed, as Revillout, Budin and quite recently Davet have shown."

Whitridge Williams says (Obstetrics, 2nd edition, 1908, p. 668): "Fxceptionally tetanic contractions of the levator ani muscle may 
seriously interfere with the descent of the head. In this condition, which is analogous to the vaginismus of non-pregnant women, a thick ring-like structure completely encircles and markedly constricts the vagina several centimetres above the vulva. Hue, in 1906, collected a number of such cases.

"Ordinarily the condition yields to the administrations of sedatives or anasthetics, though in one of my patients the obstruction persisted in spite of profound anæsthesia, and it was only after steady pressure had been exerted upon it for some minutes that it relaxed sufficiently to permit the passage of the hand folded in the shape of a cone."

Eden says (Obstetrics, 1906, p. 296): "In elderly primipara (over thirty) the perineal body and other tissues composing the pelvic floor appear to be deficient in elasticity, and consequently dilatation of the vulva at the end of the second stage does not proceed normally. The head may therefore be delayed for a long time upon the pelvic floor, slightly distending the vuiva with each pain, but unable to escape; unless the uterine contractions are unusually powerful, forceps will be required to extract it."

Thus, too, in Jewett's Practice of Obstetrics by American Authors (1899), under the head "Dystocia," we find: "The vagina and vulva may be narrow and tough in elderly primipara, in very muscular women, and in conditions of cicatricial contraction after previous injury."

It is therefore evident that the muscular floor of the pelvis does sometimes prevent or delay the expulsion of the foetal head, and in my experience this occurs not infrequently. Further, and this is more important to recognize, in this obstruction we have the most frequently occurring and most potent factor concerned in the etiology of prolapse; for this obstruction can only be overcome either by a dilatation or a tearing of the muscle (post rectal fibres of the pubo-rectalis). It is in this laceration that prolapse finds its possibility in the vast majority of cases.

As regards the retention of frecal masses, it is significant to notice that constipation is much more frequent in the female sex, in whom the pubo-rectalis muscle is better developed than in the male. In both sexes it is well known that freal masses can be easily retained within the bowel, even in the presence of the desire to defrcate, by a voluntary control. This consists in the active contraction of the levator ani muscle, and more especially of the pubo-rectalis, which encircling the rectum posteriorly in the bend of its perineal flexure, draws this part of the gut forwards to the symphysis, thus increasing the curvature and tending to obliterate the lumen; indeed Holl for this reason called the muscle the sphincter of the rectum. This supposition is enforced by the knowledge that when the desire to 
defacate is exceedingly strong, delay can only be obtained by calling forth contractions not only of the levator ani, but also by extraordinary movements of certain of the muscles of the lower extremities, amongst which the obturator internus probably plays a considerable part, since it forms a large bulk of tissue upon the lateral pelvie wall which will bulge considerably inwards on contraction and so offer a mechanical obstruction.

Thus the Prof. Schäfer writer (Physiology, vol. ii, p. 335, 1900): "When the frees have reached the rectum, the passing is still kept in check by the tonic contraction of the two sphincters of the anus, the internal sphincter composed of involuntary and the external sphincter of voluntary muscle. When the contents of the rectum are fluid, or when they are forced against the sphincters by strong peristaltic contractions of the lower bowel, the action of the sphincters may bo voluntarily reinforced by active contractions of the levator ani muscles, which raise and compress the lower segment of tho rectum or anal canal."

Landois says (Physiol., 1904, p. 284): "When the desire for stool is marked the closure of the anus can be made more secure by pressure from without through forcible rotation of the thighs ontwards and the action of the gluteal muscles."

Starling says (Physiol., 1907, p. 375): The faces "are also kept back by the acute angle that the last part of the rectum makes with the preceding part, and by the contraction of the perineal muscles which maintain this curvature and empty the lower part of the bowel."

It is therefore evident that defreation can be delayed by tho active voluntary contraction of the levator ani musele.

\section{As regards the Tntra-abdominal Pressure.}

The same authority ${ }^{1}$ has said that: "To mention intra-abdominal pressure as a cause of prolapse was like mentioning gravitation as a cause of falling through a broken plank - -true, in a sense, but uninteresting." But the condition of prolapse of the uterus is not like that of a body falling through a broken plank, nor like that of a body falling through a hole in the floor; but it is entirely due to an increased pressure within the abdominal cavity, and does not occur when the intra-abdominat pressure is low, wen when the deficiency in the pelvie floor is present, e.g., during rest in the recumbent position. If the question of gravity had alone to be considered it were possible for connective tissue of the slenderest kind to support the uterus, a weight of $1 \frac{1}{2}$ ounces only; but it is because the pressure

1. Dr. Fothergill, ibid, p. 219. 
downwards is so frequently excessively raised that the connective tissue attachments elongate when the muscle beneath is unable, hecause impaired, to oppose and to resist the pressure from above.

Since, when the pelvic floor is defective, prolapse only results when the intra-abdominal pressure is sufficiently raised, it is obvious that the increase of this pressure is of determining importance in the occurrence of prolapse. This is the reason that, whilst the poor are so prone to this dystrophy the rich so frequently escape; in both the injury to the pelvic floor is the same, but the poor cannot obtain sufficient rest and cannot avoid often long continued and excessive strains (i.e., increased intra-abdominal pressure), whilst the rich can enjoy a surfeit of recumbency and are able to avoid the injurious downthrust of the viscera upon the weakened and impaired pelvic floor. In view of this all important determining influence, exerted by the intra-abdominal pressure, it is strange to find an author regarding it as 'true in a sense but uninteresting'. . . . So far from being uninteresting, it is of predominating importance and interest, for without a due recognition of the presence, causation and magnitude of this intra-ahdominal pressure, it is certain the processes which determine the staties of the viscera cannot be understood.

The lowering of this intra-abdominal pressure, and the relief which this brings to the overwrought and impaired pelvic floor, is one of the most potent factors in the prevention and treatment of prolapse. There is no doubt it plays a vast, yet unobtrusive, and even yet unrecognized influence in the results achieved in the operative treatment of prolapse. It plays its silent yet all important part in the good results attained in all and each of the various operative procedures, but the success of each individual mothod is ascribed to the details of that particular method alone, and in this way each ligament in turn has been regarded as the chief support of the uterus because prolapse has been benefited or cured by operating upon the ligament in question, no tribute to natural reparative processes being allowed. Who can doubt the enormous benefit derived by a fortnight's or a three wecks' rest in bed? during which period of recumbency and absence of mental and physical strain, the intra-abdominal pressure is reduced to a minimum, and the overstretched and injured pelvic floor has a chance to return to its normal tone and its usual position. The benefit of such treatment with quietness, sufficient sleep and appropriate dieting, especially if combined with massage, is incalculable.

I cannot enter further here into this question, interesting as it is. The importance of the intra-abdominal pressure as a factor in determining and in maintaining the position of the uterus and other pelvic viscera must, I think, be obvious to everyone. On the other hand, the pelvic floor musculature is of equal importance, for whilst the former determines prolapse, deficiency of the latter predisposes to 
it; nor does this dystrophy occur except in uncommon and unusual cases when this museulature has not been injured.

The more one enters into this question the more convinced one becomes of the validity of this conception. From whatever aspect we view it, the same great principles stand out in marked and conspicnous relief. The study of the normal, whether of structure or of function; the study of disease, whether of its causation or effect; the study of treatment, whether in the direction of prevention or of cure, all point to it. These mute signposts, dumb as they are, are eloquent enough will we but look at them; we can no longer neglect them, nor remain insensible to their teaching. 